\title{
REVISED Evaluation of electrochemiluminescence
}

\section{immunoassays for immunosuppressive drugs on the Roche}

\section{cobas e411 analyzer [version 2; peer review: 2 approved, 1}

\section{approved with reservations]}

\author{
Angela W.S. Fung (iD1,2*, Michael J. Knauer (D1,3*, Ivan M. Blasutig1,4, \\ David A. Colantonio ${ }^{1,5}$, Vathany Kulasingam (Di) 1,6 \\ ${ }^{1}$ Department of Laboratory Medicine and Pathobiology, University of Toronto, Toronto, ON, M5S 1A8, Canada \\ 2Department of Pathology and Laboratory Medicine, St. Paul's Hospital, Vancouver, BC, V6Z 1Y6, Canada \\ ${ }^{3}$ Lifelabs Medical Laboratories, Toronto, ON, M9W 6J6, Canada \\ ${ }^{4}$ Division of Biochemistry, Children's Hospital of Eastern Ontario, Ottawa, ON, K1H 8L1, Canada \\ ${ }^{5}$ Department of Pediatric Laboratory Medicine, The Hospital for Sick Children, Toronto, ON, M5G 1X8, Canada \\ ${ }^{6}$ Department of Clinical Biochemistry, University Health Network, Toronto, ON, M5G 2C4, Canada \\ * Equal contributors
}

V2 First published: 13 Oct 2017, 6:1832

https://doi.org/10.12688/f1000research.12775.1

Latest published: 12 Dec 2017, 6:1832

https://doi.org/10.12688/f1000research.12775.2

\section{Abstract}

Background: Therapeutic drug monitoring of immunosuppressant drugs are used to monitor drug efficacy and toxicity and to prevent organ transplant rejection. This study evaluates the analytical performance of semi-automated electrochemiluminescence immunoassays (ECLIA) for cyclosporine (CSA), tacrolimus (TAC) and sirolimus (SRL) on the Roche cobas e 411 analyzer at a major transplant hospital to assess method suitability and limitations. Methods: Residual whole blood samples from patients undergoing immunosuppressant therapy were used for evaluation. Imprecision, linearity, functional sensitivity, method comparisons and lot-to-lot comparisons were assessed.

Results: Total imprecision ranged from 3.3 to $7.1 \%$ for CSA, 3.9 to 9.4\% for TAC, and 4.6 to $8.2 \%$ for SRL. Linearity was verified from 30.0 to $960.9 \mu \mathrm{g} / \mathrm{L}$ for CSA, from 1.1 to $27.1 \mu \mathrm{g} / \mathrm{L}$ for TAC, and from 0.5 to $32.3 \mu \mathrm{g} / \mathrm{L}$ for $\mathrm{SRL}$. The functional sensitivity met the manufacturer's claims and was determined to be $<6.5 \mu \mathrm{g} / \mathrm{L}$ for CSA, $1.1 \mu \mathrm{g} / \mathrm{L}$ for TAC, and $<0.1 \mu \mathrm{g} / \mathrm{L}$ for $\mathrm{SRL}(\mathrm{CV} \leq 20 \%)$. Deming regression analysis of method comparisons with the ARCHITECT immunoassay yielded slopes of 0.917 (95\% Cl: 0.885-0.949) and $r$ of 0.985 for CSA, 0.938 (95\% $\mathrm{Cl}: 0.895-0.981)$ and $r$ of 0.974 for TAC, and $0.842(0.810-1.110)$ and $r$ of 0.982 for SRL. Deming regression analysis of comparisons with the LC-MS/MS method yielded slopes of 1.331 (95\% Cl: 1.167-1.496) and $r$ of

\section{Open Peer Review}

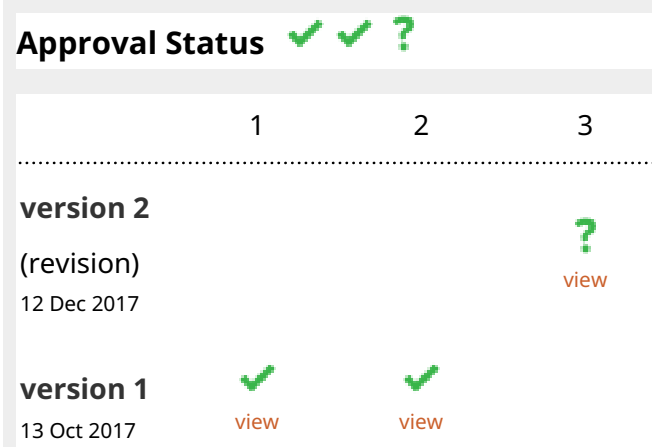

1. Jennifer Shea, Horizon Health Network, Saint John, Canada

2. Julie Shaw, University of Ottawa, Ottawa, Canada

\section{Stephen A. Hill, McMaster University, \\ Hamilton, Canada}

Any reports and responses or comments on the article can be found at the end of the article. 
0.969 for CSA, 0.924 (95\% Cl: $0.843-1.005)$ and $r$ of 0.984 for TAC, and

0.971 (95\% Cl: $0.913-1.030)$ and $r$ of 0.993 for SRL.

Conclusions: The cobas e 411 ECLIA for CSA, TAC, and SRL have acceptable precision, linearity, and functional sensitivity. The method comparisons correlated well with the ARCHITECT immunoassay and LC-MS/MS and is fit for therapeutic drug monitoring

\section{Keywords}

Cyclosporine, tacrolimus, sirolimus, immunoassay, ECLIA

Corresponding author: Vathany Kulasingam (vathany.kulasingam@uhn.ca)

Author roles: Fung AWS: Data Curation, Formal Analysis, Investigation, Methodology, Validation, Visualization, Writing - Original Draft Preparation, Writing - Review \& Editing; Knauer MJ: Data Curation, Formal Analysis, Investigation, Methodology, Visualization, Writing Original Draft Preparation, Writing - Review \& Editing; Blasutig IM: Conceptualization, Project Administration, Writing - Review \& Editing; Colantonio DA: Methodology, Writing - Review \& Editing; Kulasingam V: Conceptualization, Data Curation, Formal Analysis, Investigation, Methodology, Project Administration, Resources, Software, Supervision, Validation, Writing - Original Draft Preparation, Writing - Review \& Editing

Competing interests: No competing interests were disclosed.

Grant information: The author(s) declared that no grants were involved in supporting this work.

Copyright: ( 2017 Fung AWS et al. This is an open access article distributed under the terms of the Creative Commons Attribution License, which permits unrestricted use, distribution, and reproduction in any medium, provided the original work is properly cited. Data associated with the article are available under the terms of the Creative Commons Zero "No rights reserved" data waiver (CC0 1.0 Public domain dedication).

How to cite this article: Fung AWS, Knauer MJ, Blasutig IM et al. Evaluation of electrochemiluminescence immunoassays for immunosuppressive drugs on the Roche cobas e411 analyzer [version 2; peer review: 2 approved, 1 approved with reservations] F1000Research 2017, 6:1832 https://doi.org/10.12688/f1000research.12775.2

First published: 13 Oct 2017, 6:1832 https://doi.org/10.12688/f1000research.12775.1 


\section{REVISED Amendments from Version 1}

This revised manuscript incorporates the LC-MS/MS method description from the "Supplementary Materials" section into the main text's "Methods" section for easier access. A brief discussion on the limitation of an imprecision study that was performed using a single reagent lot, and on the lack of standardization for immunosuppressant drugs are now included. The calibration frequency and edits for grammatical errors have been incorporated.

\section{See referee reports}

\section{Introduction}

Immunosuppressive drugs (ISD), such as the calcineurin inhibitors (cyclosporine (CSA) and tacrolimus (TAC)) and mammalian target of rapamycin (mTOR) inhibitors (sirolimus (SRL) and everolimus), are critical to the maintenance of solid organ transplantation ${ }^{1}$. CSA is a cyclic undecapeptide and TAC (also known as FK-506) is a macrolide lactone. CSA binds to cyclophilin A/B and inhibits calcineurin. TAC binds to FK506-binding protein 12 (FKBP-12) to form the calcineurin inhibitory complex. Inhibition of calcineurin, a serine/threonine phosphatase, leads to altered calcium-dependent signal transduction, and decreases T-cell activation and downregulates anti-inflammatory responserelated genes ${ }^{2,3}$. SRL (also known as rapamycin) is a 31-membered macrolide antibiotic that binds to FKBP-12 and allosterically targets the mTOR pathway, inhibiting cell cycle progression, T-cell proliferation and differentiation ${ }^{2}$. SRL has structural similarities to TAC and competes with TAC for FKBP-12 binding ${ }^{1,2}$. All three ISDs are characterized by having variable absorption, poor bioavailability, strong affinity to blood proteins, leukocytes, and/or erythrocytes, and metabolism via cytochrome CYP3A4/5 and efflux transport by P-glycoprotein ${ }^{2}$.

Therapeutic drug monitoring is a mainstay in immunosuppressant therapy. ISDs have narrow therapeutic ranges ${ }^{2}$, high interindividual variability in pharmacokinetics and pharmacogenetics ${ }^{4,5}$, susceptibility to food- and drug-drug interactions ${ }^{6}$, and adverse consequences if plasma drug levels are not maintained ${ }^{5,7}$. Similar toxic effects have been described for CSA and TAC, due to their overlapping mechanism of action, and includes nephrotoxicity, hypertension, and neurotoxicity ${ }^{2}$. TAC is a more potent calcineurin inhibitor than CSA, due to increased affinity for FKBP-12 and the advantage of decreased nephrotoxicity, risk of hyperlipidemia and hypertension ${ }^{1,2,8}$. TAC, however, is more likely to cause posttransplantation diabetes ${ }^{1,9,10}$. SRL does not cause renal toxicities; however, long-term SRL use can induce leukopenia, thrombocytopenia, and dyslipidemia ${ }^{11,12}$. The target therapeutic range for each ISD may vary depending on type of organ transplanted, time from transplantation, co-administered drugs, and method of analysis.

Recently, semi-automated electrochemiluminescence immunoassays (ECLIA) for the quantification of CSA, TAC, and SRL in whole blood were developed and made available by Roche Diagnostics (GmbH, Mannheim, Germany) $)^{13,14}$. In this study, we evaluated the analytical performance of ECLIA method for CSA, TAC, and SRL on the Roche cobas e411 analyzer and compared to the commonly used chemiluminescent microparticle immunoassay (CMIA) method on the Abbott ARCHITECT i2000 analyzer (Abbott Laboratories, Abbott Park, IL, USA). This is the first report on the evaluation of ECLIA SRL, and compares the performance of all three ISDs together.

\section{Methods}

Specimen source and handling

Ethics approval for this study was waived by the Research Ethics Board at the University Health Network in Toronto, Ontario, Canada (16-6312) for use of routine collected specimens for the evaluation of method performance. Residual EDTA whole blood specimens from 300 patients undergoing immunosuppressant therapy (either cyclosporine, tacrolimus, or sirolimus) at the University Health Network, and CAP proficiency testing samples were used in this evaluation. Samples were collected and analyzed by the Abbott CMIA within the same day, then stored as per manufacturer recommendations and analyzed later by the Roche ECLIA and LC-MS/MS methods. Samples were thawed and equilibrated to room temperature for 30 minutes and mixed well prior to analysis. In accordance with stability studies on whole blood ISD specimens, samples were analyzed within three months of collection and did not undergo more than two freeze-thaw cycles $^{15-18}$.

\section{Electrochemiluminescence immunoassay (ECLIA) method on Roche cobas e 411}

The cobas ECLIAs (Roche Diagnostics GmbH, Mannheim, Germany) for CSA, TAC, and SRL are based on the competition of analyte in sample with a ruthenium-labeled analogue. A voltage is applied and electrochemiluminescence signal is detected. Testing was performed according to the manufacturer's instructions. Briefly, the samples (calibrators, QC, whole blood samples) were manually pretreated by combining $300 \mu \mathrm{L}$ of whole blood with $300 \mu \mathrm{L}$ of Universal ISD Sample Pretreatment Reagent (containing zinc sulfate and methanol) and vortexed for 10 seconds to lyse the red blood cells, precipitate proteins and extract the analyte. The samples were centrifuged for 4 minutes at $15,000 \mathrm{x} \mathrm{g}$, and the supernatant was decanted for analysis. Analysis was performed within 30 minutes of preparation to prevent evaporation of the extracted samples. The ECLIA assays were calibrated as per manufacturer's instruction by a 2-point calibration using calibrators traceable to pure standard materials reconstituted in whole blood matrix by gravimetrical methods.

\section{Chemiluminescent microparticle immunoassay (CMIA) method on Abbott ARCHITECT i2000}

The ARCHITECT CMIAs (Abbott Laboratories, Abbott Park, IL, USA) for CSA, TAC, and SRL are based on competition of analyte in sample with acridinium-labeled analogue. The samples were manually pretreated according to the manufacturer's instructions and site-specific standard operating procedures. For CSA, $100 \mu \mathrm{L}$ of Cyclosporine Solubilizing Reagent (4\% saponin) and $400 \mu \mathrm{L}$ of Cyclosporine Precipitation Reagent (zinc sulfate in methanol and ethylene glycol) was added to $200 \mu \mathrm{L}$ of sample $^{19,20}$. For TAC, $200 \mu \mathrm{L}$ of sample was mixed with $200 \mu \mathrm{L}$ of Tacrolimus Precipitation Reagent (zinc sulfate in methanol) ${ }^{21,22}$. For SRL, $150 \mu \mathrm{L}$ of sample was mixed with $300 \mu \mathrm{L}$ of Sirolimus 
Precipitation Reagent (zinc sulfate in $>50 \% \mathrm{v} / \mathrm{v}$ DMSO and ethylene glycol), vortexed and heated at $42^{\circ} \mathrm{C}$ for 10 minutes ${ }^{23,24}$. All ISD samples were then vortexed for 10 seconds and centrifuged for 4 minutes at $15,000 \mathrm{x}$ g. The supernatants were decanted into labelled tubes and assayed within 30 minutes of sample preparation. The ARCHITECT CMIAs are calibrated according to the sitespecific standard operation procedures and manufacturer's instructions, with a 6-point 4-parameter logistic curve fit (4PLC, $\mathrm{y}$-weighted) that is traceable to pure standard materials in a whole blood matrix by gravimetrical methods. Internal QC was evaluated with Bio-Rad Lyphochek Whole Blood ISD Controls levels 1,3 , and 4 .

\section{Electrospray ionization liquid chromatography tandem mass spectrometry (ESI-LC-MS/MS) method}

The ESI-LC-MS/MS MRM method for CSA, TAC, and SRL were analyzed on a 4000 QTrap mass spectrometer (SCIEX) at the Hospital for Sick Children (Toronto, ON, Canada). Samples were pretreated by mixing $40 \mu \mathrm{L}$ of sample with $100 \mu \mathrm{L}$ of sample pretreatment reagent consisting of $0.04 \mathrm{M}$ zinc sulfate, and internal standards $100.0 \mu \mathrm{g} / \mathrm{L}$ cyclosporine $\mathrm{D}$ and $10.0 \mu \mathrm{g} / \mathrm{L}$ ascomycin in methanol. Samples were vortexed and centrifuged for 5 minutes at $15,000 \mathrm{x}$ g to obtain the supernatant for analysis. The analyte is separated by liquid chromatography (Nexera X2 Shimadzu) with a reverse phase $\mathrm{C}_{18}$ column (Phenomenex, $4 \times 3.0 \mathrm{~mm}$ at $45^{\circ} \mathrm{C}$ ) and gradient elution from $100 \%$ B to $50 \%$ B (Buffer A: $2 \mathrm{mM}$ ammonia acetate and $0.1 \%$ formic acid in water and Buffer B: $2 \mathrm{mM}$ ammonia acetate and $0.1 \%$ formic acid in methanol) at a flowrate of $650 \mu \mathrm{L} / \mathrm{min}$ and electrospray ionization into the mass spectrometer. The following precursor/production pairs in positive ion mode were used 1220.8/1203.8 $\mathrm{m} / \mathrm{z}$ for CSA, 821.5/768.5 m/z for TAC, and $931.6 / 864.5 \mathrm{~m} / \mathrm{z}$ for SRL. CSA and TAC were calibrated with a 6-point calibration curve using Emit 2000 CSA or TAC specific calibrators (Syva Company, Siemens Healthcare). SRL was calibrated with a 6-point calibration curve using 6Plus1 Multilevel immunosuppressant calibrators (Chromsystems). There is generally a lack of certified reference materials for TDMrelevant drugs, including the ISDs. There is currently only one ISD certified reference material for tacrolimus in whole blood (ERM-DA110a), and current efforts are directed towards standardization $^{25}$. Internal QC were evaluated with Bio-Rad Lyphochek Whole Blood ISD Controls levels 1, 3, and 4.

\section{Imprecision}

Three levels of manufacturer multi-analyte QC materials (Roche Diagnostics PreciControl ISD levels 1, 2, and 3) and third-party multi-analyte QC materials (Bio-Rad Lyphochek Whole Blood ISD Controls levels 1, 3, and 4) were analyzed. QC samples were prepared and measured in duplicate, one run per day over 10 days. The acceptance criterion for total imprecision was based on the recommendation of the International Association of Therapeutic Drug Monitoring and Clinical Toxicology
(IATDMCT) expert consensus group of $\leq 10 \%{ }^{25}$.

\section{Functional sensitivity}

Residual patient sample with levels 2-3 times the claimed limit of quantification (LoQ) was used to generate a series of dilutions with blank whole blood. The neat sample and dilutions were measured in triplicates within one day. The precision profile curve was used to calculate the LoQ concentration corresponding to a CV of $20 \%$ with the upper $95 \%$ confidence limit.

\section{Linearity}

Since there is a lack of elevated CSA and TAC patient specimen, CSA and TAC linearity were assessed using CAP EQA linearity materials (6 concentrations measured in duplicate). SRL linearity was assessed using a patient sample above the upper measuring range diluted with blank whole blood to 6 concentrations and measured in duplicate. The acceptance criterion was defined as slope of $1.00 \pm 0.05$ and deviation $<10 \%$.

\section{Method comparison}

Method comparison experiments were assessed where anonymized residual patient samples spanning the analytical measuring range for each analyte were measured once per method. CSA samples concentrations ranged from 41.0 to $1808.0 \mu \mathrm{g} / \mathrm{L}$, TAC ranged from 2.1 to $30.0 \mu \mathrm{g} / \mathrm{L}$, and SRL ranged from 1.8 to $34.6 \mu \mathrm{g} / \mathrm{L}$ as determined by ARCHITECT CMIA. Roche ECLIA measurements were compared to ARCHITECT CMIA ( $n=100)$. To further elucidate the accuracy between immunoassays, a subset of samples was also analyzed by LC-MS/MS $(n=20)$. Lot-to-lot assessment was also performed between two lots of reagents for each ISD $(n=20)$. The slope, intercept, correlation coefficient $r$ were analyzed by Deming regression analysis. The acceptance criteria for method comparison were defined as a slope of $1.00 \pm 0.15$ and $r$ of $\geq 0.95$, meanwhile for lot-to-lot comparison were defined as a slope of $1.00 \pm 0.05$ and $r$ of $\geq 0.95$.

\section{Statistical analysis}

Microsoft Excel (version 1708, Microsoft Office) and/or EP Evaluator (version 7.0.0.307, Data Innovations) were used for statistical analysis.

\section{Results and discussion}

To assess imprecision, three levels of manufacturer (Roche PreciControl) and third-party (Bio-Rad Lyphochek) multi-analyte QC materials were analyzed using one lot of reagents in duplicate, one run per day over 10 days (Table 1). For the PreciControl, the total imprecision was $<7.1 \%$ for CSA, $<9.4 \%$ for TAC, and $<5.6 \%$ for SRL. Imprecision for CSA and TAC were comparable to other studies ${ }^{13,14}$. Our study additionally evaluated third-party QC performance on ECLIA ISD assays, a total imprecision of $<4.7 \%$ for CSA, $<6.3 \%$ for TAC, and $<8.2 \%$ for SRL were determined. The imprecision goal of $\leq 10 \%$, based on the rec- 


\section{Table 1. Total imprecision for cyclosporine, tacrolimus, and sirolimus determined by the ECLIA method (duplicate per run, 1 run per day for 10 days).}

\begin{tabular}{|c|c|c|c|}
\hline & Samples & $\begin{array}{l}\text { Mean Conc. } \\
(\mu \mathrm{g} / \mathrm{L})^{*}\end{array}$ & $\begin{array}{r}\text { Total CV } \\
(\%)\end{array}$ \\
\hline \multirow{6}{*}{$\begin{array}{r}\text { Cyclosporine } \\
\text { (CSA) }\end{array}$} & Roche ISD L1 & 63.1 & 7.1 \\
\hline & Roche ISD L2 & 271.1 & 5.1 \\
\hline & Roche ISD L3 & 976.9 & 3.8 \\
\hline & Bio-Rad WB ISD L1 & 67.3 & 4.7 \\
\hline & Bio-Rad WB ISD L3 & 346.5 & 4.5 \\
\hline & Bio-Rad WB ISD L4 & 740.1 & 3.3 \\
\hline \multirow{6}{*}{$\begin{array}{r}\text { Tacrolimus } \\
\text { (TAC) }\end{array}$} & Roche ISD L1 & 2.5 & 9.4 \\
\hline & Roche ISD L2 & 9.2 & 6.9 \\
\hline & Roche ISD L3 & 16.9 & 4.1 \\
\hline & Bio-Rad WB ISD L1 & 4.1 & 6.3 \\
\hline & Bio-Rad WB ISD L3 & 7.8 & 5.3 \\
\hline & Bio-Rad WB ISD L4 & 15.2 & 3.9 \\
\hline \multirow{6}{*}{$\begin{array}{r}\text { Sirolimus } \\
(\mathrm{SRL})\end{array}$} & Roche ISD L1 & 3.4 & 4.6 \\
\hline & Roche ISD L2 & 8.7 & 5.6 \\
\hline & Roche ISD L3 & 15.4 & 4.7 \\
\hline & Bio-Rad WB ISD L1 & 5.9 & 4.7 \\
\hline & Bio-Rad WB ISD L3 & 9.5 & 5.7 \\
\hline & Bio-Rad WB ISD L4 & 14.2 & 8.2 \\
\hline
\end{tabular}

${ }^{*}$ Conventional unit: $1 \mu \mathrm{g} / \mathrm{L}=1 \mathrm{ng} / \mathrm{mL}$

ommendation of the IATDMCT expert consensus group, was achieved for all QC samples ${ }^{25}$. Note that this imprecision study was performed using a single reagent lot and may not represent variations due to other variables such as changes in operator, calibrator and reagent lots, and ambient operating conditions. Overall, the ECLIA methods demonstrate acceptable precision.

The ECLIA methods offer a wider linear analytical measuring range for CSA and TAC than CMIA methods. ECLIA CSA, TAC, and SRL were linear up to $960.9 \mu \mathrm{g} / \mathrm{L}, 27.1 \mu \mathrm{g} / \mathrm{L}$, and $32.3 \mu \mathrm{g} / \mathrm{L}$, respectively. The higher upper limit allows TDM and pharmacokinetic analysis of ISD at different time points and peak concentrations offering additional flexibility ${ }^{26,27}$.

The claimed functional sensitivity of the ECLIA ISD methods are improved for TAC and SRL compared to CMIA ISD methods. The functional sensitivities were assessed and the precision profile was used to calculate the LoQ corresponding to a $\mathrm{CV}$ of
$20 \%$ with upper $95 \%$ confidence limit. The functional sensitivities were determined to be $<6.5 \mu \mathrm{g} / \mathrm{L}$ for CSA, $1.1 \mu \mathrm{g} / \mathrm{L}$ for TAC, and $<0.1 \mu \mathrm{g} / \mathrm{L}$ for SRL, which meets the 2007 European consensus guideline and IATDMCT expert consensus group recommended LoQ of $20.0 \mu \mathrm{g} / \mathrm{L}$ for CSA and a LoQ of $1.0 \mu \mathrm{g} / \mathrm{L}$ for both TAC and SRL ${ }^{25,28}$.

For method comparison, anonymized residual patient samples spanning the analytical measuring range for each analyte were measured. CSA samples concentrations ranged from 41.0 to $1808.0 \mu \mathrm{g} / \mathrm{L}$, TAC ranged from 2.1 to $30.0 \mu \mathrm{g} / \mathrm{L}$, and SRL ranged from 1.8 to $34.6 \mu \mathrm{g} / \mathrm{L}$ as determined by CMIA. ECLIA ISDs measurements were compared to CMIA ISDs $(n=100)$. The acceptance criteria were defined as a slope of $1.00 \pm 0.15$ and $r$ of $\geq 0.95$. Figure 1 shows the Deming regression and BlandAltman analysis for CSA, TAC, and SRL. ECLIA and CMIA CSA (Figure 1A) showed good agreement with a slope of 0.917 (95\% CI: 0.885-0.949), intercept of -15.2 (95\% CI: -39.4-9.0), and $r$ of 0.985 . For TAC, the ECLIA TAC also showed good agreement with CMIA TAC (Figure 1B) with a slope of 0.938 (95\% CI: 0.895-0.981), intercept of 0.2 (95\% CI -0.4-0.8), and $r$ of 0.974 . Similar trends were observed by others (slopes of 0.87 for CSA, and $0.96-0.98$ for TAC) $)^{14,29}$. Reported for the first time, method comparison of ECLIA and CMIA SRL (Figure 1C) showed a slope of 0.842 (95\% CI: 0.810-1.110), intercept of 0.9 (95\% CI: 0.4-1.4), and $r$ of 0.982. Overall, all three ECLIA ISDs met our acceptance criteria, with SRL slightly exceeding the limit for the slope.

To further examine ECLIA performance with CMIA, a subset of samples was also analyzed by LC-MS/MS $(n=20)$. For both CSA immunoassays, a positive bias was observed when compared with LC-MS/MS, similarly observed by others ${ }^{13,29,30}$ (Figure S1). For TAC, both the ECLIA and CMIA TAC had good agreement with LC-MS/MS, also similarly observed by others for different cohorts of solid organ transplant ${ }^{14,21,29}$ (Figure S2). Reported for the first time, the ECLIA SRL compared to LC-MS/MS showed a slope of 0.971 (95\% CI: 0.913-1.030), intercept of 2.4 (95\% CI: 1.6-3.3), and $r$ of 0.993 (Figure 2). Meanwhile, CMIA SRL compared to LC-MS/MS showed a slope 1.119 (95\% CI: 1.051-1.187), intercept of 1.4 (95\% CI: 0.5-2.4), and $r$ of 0.993. Based on our small sample size, both ECLIA and CMIA SRL generally showed good correlation with LC-MS/MS, with ECLIA SRL with better agreement to LC-MS/MS.

For lot-to-lot comparisons, Deming regression analysis of 20 residual patient samples tested by 2 lots of reagent for CSA shows a slope of 0.998 (95\% CI: 0.967-1.029), intercept of $8.5(95 \%$ CI: -10.2-27.2), and $r$ of 0.998. Lot-to-lot comparison for TAC shows a slope of 0.972 (95\% CI: 0.936-1.008), intercept of -0.4 (95\% CI: -0.8-0.0), and $r$ of 0.997. And lot-to-lot comparison for SRL shows a slope of 0.913 (95\% CI: 0.841-0.985), intercept 

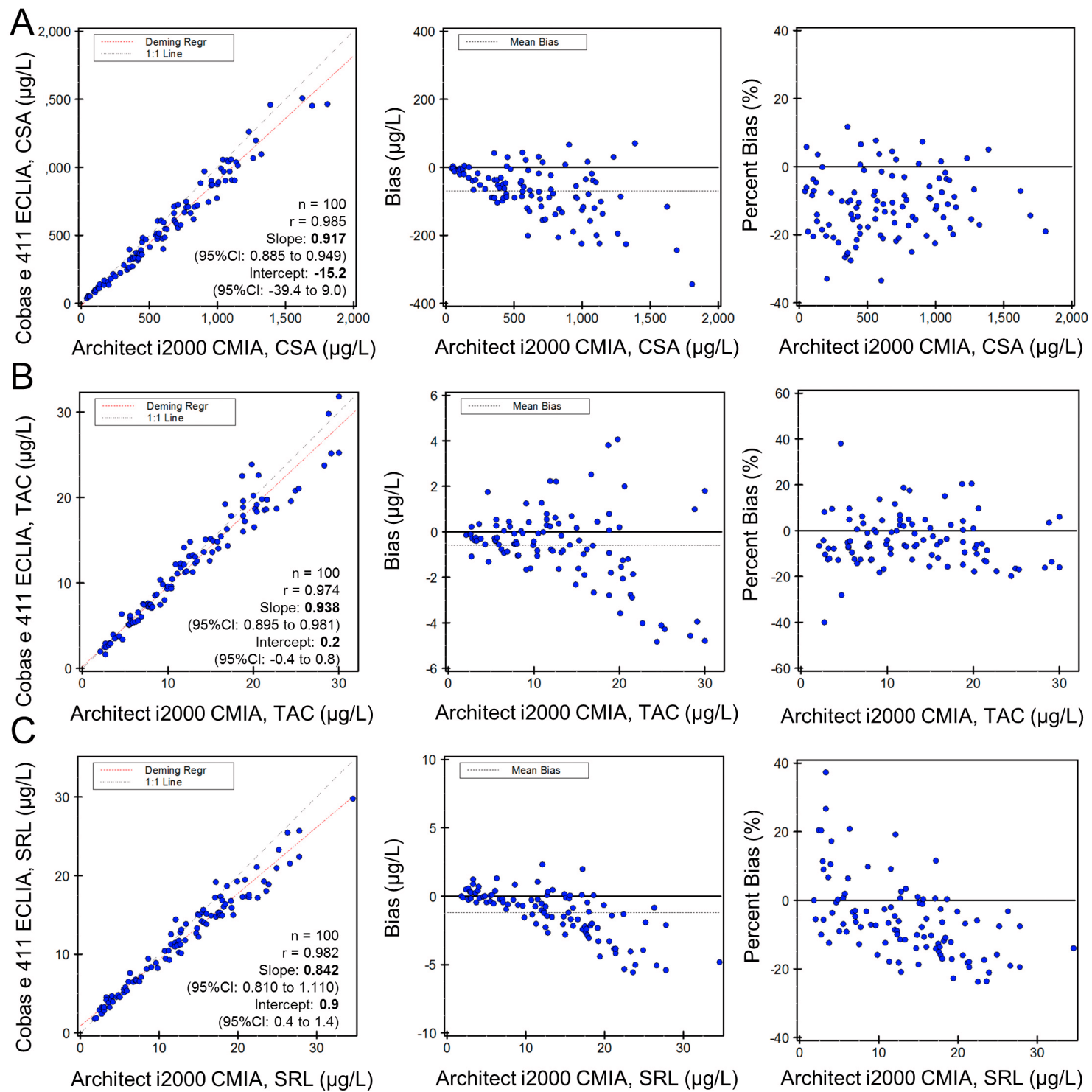

Figure 1. Method comparisons and Bland-Altman plots for (A) cyclosporine (CSA), (B) tacrolimus (TAC), and (C) sirolimus (SRL) between cobas e411 ECLIA and ARCHITECT i2000 CMIA.

of 0.1 (95\% CI: -0.9-1.2), and $r$ of 0.988. All three ISDs had good correlation between 2 different lots of reagents.

Evaluation on practical considerations included ease-of-use, throughput, and workflow of the method. The sample pretreatment for the ECLIA method is faster, simpler, and more convenient than CMIA method due to the use of a single universal sample pretreatment reagent and protocol for all three ISDs. Additionally, there is no heating step for the SRL ECLIA method, which leads to a simpler workflow. The ECLIA universal sample pretreatment reagent and protocol would enable better workflow, simpler sample handling and inventory control. The ECLIA method has an assay time of 18 minutes compared to CMIA of 30 minutes. Both ECLIA and CMIA have a lot calibration stability of approximately one month, thus requiring similar calibration frequency. Together, the needs of the individual clinical laboratory will dictate whether some of these practical considerations play a role in the method selection. 
A
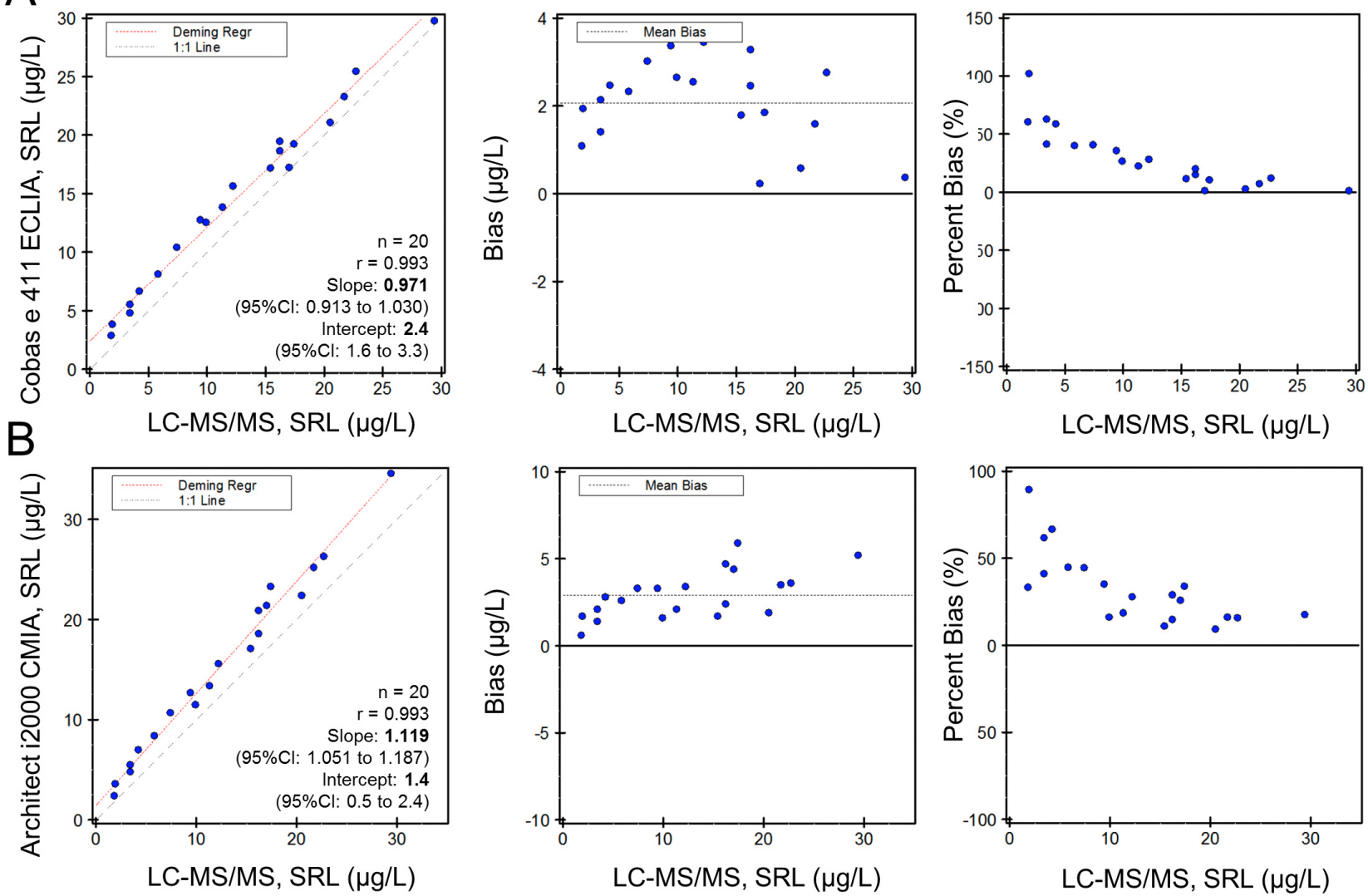

Figure 2. Method comparisons and Bland-Altman plots for sirolimus (SRL) between cobas e411 ECLIA and LC-MS/MS.

\section{Dataset 1. File containing the raw data and a table of contents for} the data file

http://dx.doi.org/10.5256/f1000research.12775.d180033

\section{Conclusion}

In conclusion, the overall analytical evaluation of the ECLIA method for CSA, TAC, and SRL met acceptable performance. ECLIA CSA showed better precision than our current CMIA CSA. ECLIA CSA and TAC showed better linearity range, and ECLIA TAC and SRL showed better functional sensitivity than CMIA methods. Method comparisons showed good correlations and agreement between ECLIA ISDs and CMIA ISDs.

\section{Data availability}

Dataset 1: File containing the raw data and a table of contents for the data file. doi, 10.5256/f1000research.12775.d180033 ${ }^{31}$
Competing interests

No competing interests were declared.

Grant information

The author(s) declared that no grants were involved in supporting this work.

\section{Acknowledgements}

We thank the technologists and support staff at University Health Network and the Hospital for Sick Children in Toronto, Ontario, Canada for their contributions to this study. The cobas ECLIA ISD reagents used in the method evaluation were provided by Roche Diagnostics.

Roche Diagnostics had no role in the design, analysis, or interpretation of this study. 


\section{Supplementary material}

Figure S1: Method comparisons and Bland-Altman plots for cyclosporine (CSA) between (A) cobas e 411 ECLIA and LC-MS/MS, and (B) ARCHITECT i2000 CMIA and LC-MS/MS.

Click here to access the data.

Figure S2: Method comparisons and Bland-Altman plots for tacrolimus (TAC) between (A) cobas e 411 ECLIA and LC-MS/MS, and (B) ARCHITECT i2000 CMIA and LC-MS/MS.

Click here to access the data.

1. Halloran PF: Immunosuppressive drugs for kidney transplantation. $N$ Engl $J$ Med. 2004; 351(26): 2715-29.

PubMed Abstract | Publisher Full Text

2. Mika A, Stepnowski $P$ : Current methods of the analysis of immunosuppressive agents in clinical materials: A review. J Pharm Biomed Anal. 2016; 127: 207-31. PubMed Abstract | Publisher Full Text

3. Oetjen E, Thoms KM, Laufer $\mathrm{Y}$, et al:: The immunosuppressive drugs cyclosporin A and tacrolimus inhibit membrane depolarization-induced CREB transcriptional activity at the coactivator level. Br J Pharmacol. 2005; 144(7): 982-93.

PubMed Abstract | Publisher Full Text | Free Full Text

4. Kurzawski M, Droździk M: Pharmacogenetics in solid organ transplantation: genes involved in mechanism of action and pharmacokinetics of immunosuppressive drugs. Pharmacogenomics. 2013; 14(9): 1099-118. PubMed Abstract | Publisher Full Text

5. Millner L, Rodriguez C, Jortani SA: A clinical approach to solving discrepancies in therapeutic drug monitoring results for patients on sirolimus or tacrolimus: Towards personalized medicine, immunosuppression and pharmacogenomics. Clin Chim Acta. 2015; 450: 15-8. PubMed Abstract | Publisher Full Text

6. Allison TL: Immunosuppressive Therapy in Transplantation. Nurs Clin North Am. 2016; 51(1): 107-20

PubMed Abstract | Publisher Full Text

7. Agrawal YP, Cid M, Westgard S, et al.: Transplant patient classification and tacrolimus assays: more evidence of the need for assay standardization. Ther Drug Monit. 2014; 36(6): 706-9.

PubMed Abstract | Publisher Full Text

8. Pham SM, Kormos RL, Hattler BG, et al:: A prospective trial of tacrolimus (FK 506) in clinical heart transplantation: intermediate-term results. J Thorac Cardiovasc Surg. 1996; 111(4): 764-72.

PubMed Abstract | Publisher Full Text | Free Full Text

9. Moore R: New-onset diabetes after renal transplantation: comparing ciclosporin and tacrolimus. Nat Clin Pract Nephrol. 2008; 4(1): 20-1. PubMed Abstract | Publisher Full Text

10. Vincenti F, Friman S, Scheuermann E, et al:: Results of an international, randomized trial comparing glucose metabolism disorders and outcome with cyclosporine versus tacrolimus. Am J Transplant. 2007; 7(6): 1506-14. PubMed Abstract | Publisher Full Text

11. Halleck F, Duerr M, Waiser J, et al:: An evaluation of sirolimus in renal transplantation. Expert Opin Drug Metab Toxicol. 2012; 8(10): 1337-56. PubMed Abstract | Publisher Full Text

12. Gummert JF, Ikonen T, Morris RE: Newer immunosuppressive drugs: a review. J Am Soc Nephrol. 1999; 10(6): 1366-80. PubMed Abstract

13. Vogeser M, Shipkova M, Rigo-Bonnin R, et al.: Multicenter analytical evaluation of the automated electrochemiluminescence immunoassay for cyclosporine. Ther Drug Monit. 2014; 36(5): 640-50. PubMed Abstract | Publisher Full Text | Free Full Text

14. Shipkova M, Vogeser M, Ramos PA, et al.: Multi-center analytical evaluation of a novel automated tacrolimus immunoassay. Clin Biochem. 2014; 47(12): 1069-77.

PubMed Abstract | Publisher Full Text

15. Holt DW, Lee T, Johnston A: Measurement of sirolimus in whole blood using high-performance liquid chromatography with ultraviolet detection. Clin Ther. 2000; 22(Suppl B): B38-48.

PubMed Abstract | Publisher Full Text

16. Capone D, Gentile A, Polichetti G, et al:: Stability of sirolimus and everolimus measured by immunoassay techniques in whole blood samples from kidney transplant patients. Int J Immunopathol Pharmacol. 2008; 21(2): 297-307. PubMed Abstract | Publisher Full Text

17. Dubbelboer IR, Pohanka A, Said R, et al:: Quantification of tacrolimus and three demethylated metabolites in human whole blood using LC-ESI-MS/MS. Ther Drug Monit. 2012; 34(2): 134-42.

PubMed Abstract | Publisher Full Text

18. Annesley TM, Hunter BC, Fidler DR, et al.: Stability of tacrolimus (FK 506) and cyclosporin G in whole blood. Ther Drug Monit. 1995; 17(4): 361-5. PubMed Abstract

19. Brate EM, Finley DM, Grote J, et al.: Development of an Abbott ARCHITECT cyclosporine immunoassay without metabolite cross-reactivity. Clin Biochem. 2010; 43(13-14): 1152-7.

PubMed Abstract | Publisher Full Text

20. Cyclosporine for Abbott Architect iSystems [package insert]. Abbott Laboratories, Abbott Park, IL USA, 2008. Reference Source

21. Bazin C, Guinedor A, Barau C, et al.: Evaluation of the Architect tacrolimus assay in kidney, liver, and heart transplant recipients. $J$ Pharm Biomed Anal. 2010; 53(4): 997-1002.

PubMed Abstract | Publisher Full Text

22. Tacrolimus for Abbott Architect iSystems [package insert]. Abbott Laboratories, Abbott Park, IL USA, 2009. Reference Source

23. Schmid RW, Lotz J, Schweigert R, et al.: Multi-site analytical evaluation of a chemiluminescent magnetic microparticle immunoassay (CMIA) for sirolimus on the Abbott ARCHITECT analyzer. Clin Biochem. 2009; 42(15): 1543-8. on the Abbott ARCHITECT analyzer. Clin
PubMed Abstract | Publisher Full Text

24. Sirolimus for Abbott Architect iSystems [package insert]. Abbott Laboratories, Abbott Park, IL USA, 2009.

Reference Source

25. Seger C, Shipkova M, Christians U, et al.: Assuring the Proper Analytical Performance of Measurement Procedures for Immunosuppressive Drug Concentrations in Clinical Practice: Recommendations of the International Association of Therapeutic Drug Monitoring and Clinical Toxicology Immunosuppressive Drug Scientific Committee. Ther Drug Monit. 2016; 38(2): Immunosu.

PubMled Abstract | Publisher Full Text

26. Oellerich M, Armstrong VW: Two-hour cyclosporine concentration determination: an appropriate tool to monitor neoral therapy? Ther Drug Monit. 2002; 24(1): 40-6.

PubMed Abstract | Publisher Full Tex 
27. Knight SR, Morris PJ: The clinical benefits of cyclosporine C2-level monitoring: a systematic review. Transplantation. 2007; 83(12): 1525-35.

PubMed Abstract | Publisher Full Text

28. Wallemacq $\mathrm{P}$, Armstrong VW, Brunet $\mathrm{M}$, et al.: Opportunities to optimize tacrolimus therapy in solid organ transplantation: report of the European consensus conference. Ther Drug Monit. 2009; 31(2): 139-52. PubMed Abstract | Publisher Full Text

29. Toole B, Gechtman C, Dreier J, et al:: Evaluation of the New Cyclosporine and Tacrolimus Automated Electrochemiluminescence Immunoassays under Field
Conditions. Clin Lab. 2015; 61(9): 1303-15.

PubMed Abstract | Publisher Full Text

30. Wallemacq P, Maine GT, Berg K, et al:: Multisite analytical evaluation of the Abbott ARCHITECT cyclosporine assay. Ther Drug Monit. 2010; 32(2): 145-51. PubMed Abstract | Free Full Text

31. Fung AWS, Knauer MJ, Blasutig IM, et al:: Dataset 1 in: Evaluation of electrochemiluminescence immunoassays for immunosuppressive drugs on the Roche cobas e411 analyzer. F1000Research. 2017.

Data Source 


\section{Open Peer Review}

\section{Current Peer Review Status:}

\section{Version 2}

Reviewer Report 03 January 2018

https://doi.org/10.5256/f1000research.14595.r29428

(C) 2018 Hill S. This is an open access peer review report distributed under the terms of the Creative Commons Attribution License, which permits unrestricted use, distribution, and reproduction in any medium, provided the original work is properly cited.

\section{Stephen A. Hill}

Department of Pathology and Molecular Medicine, McMaster University, Hamilton, ON, Canada

1. "The ECLIA methods offer a wider analytical measurement range for CSA and TAC than CMAI methods." It would be helpful to include the upper limits for the CMIA assays for comparison.

2. "The claimed functional sensitivity of the ECLAI ISD methods are improved for TAC and SRL compared to CMIA ISD methods." It would be helpful to include the CMIA data for comparison.

3. "Overall, all three ECLID ISDs met our acceptance criteria, with SRL slightly exceeding the limit for slope." The two parts of this sentence are incongruent. SRL did not meet your predetermined criteria. It is inappropriate to set pre-determined acceptance criteria and then not use them.

"In conclusion, the overall analytical evaluation of ELCIA method for CSA, TAC and SRL met acceptable performance" The same comment applies here.

One of the strengths of this paper is that pre-determined criteria were established.

I think it's fair to state the in spite of not meeting all pre-determined performance criteria, the ECLIA methods are of sufficient quality to accept them for clinical service.

Some re-wording should correct this.

Is the work clearly and accurately presented and does it cite the current literature? Yes

Is the study design appropriate and is the work technically sound? 
Are sufficient details of methods and analysis provided to allow replication by others? Yes

If applicable, is the statistical analysis and its interpretation appropriate?

Partly

Are all the source data underlying the results available to ensure full reproducibility?

Yes

Are the conclusions drawn adequately supported by the results?

Partly

Competing Interests: No competing interests were disclosed.

I confirm that I have read this submission and believe that I have an appropriate level of expertise to confirm that it is of an acceptable scientific standard, however I have significant reservations, as outlined above.

\section{Version 1}

Reviewer Report 30 November 2017

https://doi.org/10.5256/f1000research.13840.r26927

(C) 2017 Shaw J. This is an open access peer review report distributed under the terms of the Creative Commons Attribution License, which permits unrestricted use, distribution, and reproduction in any medium, provided the original work is properly cited.

\section{Julie Shaw}

Department of Pathology and Laboratory Medicine, The Ottawa Hospital, Eastern Ontario Regional Laboratories Association, University of Ottawa, Ottawa, ON, Canada

Here the authors describe evaluation of three immunosuppressant drug immunoassays on the Roche Cobas e411 analzyer. The authors evaluated the performance of assays for cyclosporine, tacrolimus and sirolimus on the system, which included imprecision, linearity and functional sensitivity studies as well as method comparisons and lot-to-lot comparisons. They compared the assay performance to the performance of the same assays on the Abbott immunoassay system and to LC MS/MS assays for the same analytes.

I have a few comments that, if the authors could address, I feel would strengthen the manuscript.

1. In the methods section, it's not clear to me whether the measurements were performed using the Roche, Abbott and LC-MS/MS methods on the same day. It is mentioned that some specimens were stored prior to analysis. Could the authors clarify this?

2. What are the immunoassays and LC-MS/MS methods traceable to? Is there a standard? This 
information would be helpful.

3. The authors mention that the Roche assays were calibrated five times over the course of the study. Is this in fitting with the manufacturer's recommendations? How does this calibration frequency compare to the Abbott system?

4. The authors should discuss the limitation of performing imprecision studies using only one lot number of reagent.

5. The authors should include a description of the LC MS/MS method that was used. There is currently no mention of this methodology until the results section.

6. There are several grammatical errors throughout the manuscript and I would recommend that the authors read it through carefully to correct these.

Is the work clearly and accurately presented and does it cite the current literature? Yes

Is the study design appropriate and is the work technically sound?

Yes

Are sufficient details of methods and analysis provided to allow replication by others? Yes

If applicable, is the statistical analysis and its interpretation appropriate?

Yes

Are all the source data underlying the results available to ensure full reproducibility? Yes

Are the conclusions drawn adequately supported by the results? Yes

Competing Interests: No competing interests were disclosed.

I confirm that I have read this submission and believe that I have an appropriate level of expertise to confirm that it is of an acceptable scientific standard.

Reviewer Report 20 October 2017

https://doi.org/10.5256/f1000research.13840.r26931

(C) 2017 Shea J. This is an open access peer review report distributed under the terms of the Creative Commons Attribution License, which permits unrestricted use, distribution, and reproduction in any medium, provided the original work is properly cited. 
Jennifer Shea

Laboratory Medicine, Saint John Regional Hospital, Horizon Health Network, Saint John, NB, Canada

Fung et al. present data evaluating three new ECLIA assays for the immunosuppressant drugs cyclosporine, tacrolimus, and sirolimus on a Roche Cobas e411 analyzer. Overall the study design is sound generally following CLSI guidelines for method validation. The introduction provides a thorough background on the drugs/assays being evaluated and the methods section clearly describes each portion of the method validation that would easily allow another researcher to replicate the study if desired. The results/discussion section succinctly presents their data in a cohesive and systematic manner and the authors do a great job at highlighting the novel aspects of their study. Their conclusion that these three new assays are fit for use for therapeutic drug monitoring is supported by the data presented.

Is the work clearly and accurately presented and does it cite the current literature? Yes

Is the study design appropriate and is the work technically sound?

Yes

Are sufficient details of methods and analysis provided to allow replication by others? Yes

If applicable, is the statistical analysis and its interpretation appropriate? Yes

Are all the source data underlying the results available to ensure full reproducibility? Yes

Are the conclusions drawn adequately supported by the results? Yes

Competing Interests: No competing interests were disclosed.

I confirm that I have read this submission and believe that I have an appropriate level of expertise to confirm that it is of an acceptable scientific standard. 
The benefits of publishing with F1000Research:

- Your article is published within days, with no editorial bias

- You can publish traditional articles, null/negative results, case reports, data notes and more

- The peer review process is transparent and collaborative

- Your article is indexed in PubMed after passing peer review

- Dedicated customer support at every stage

For pre-submission enquiries, contact research@f1000.com 